\title{
Interactions cellulaires dans le foie
}

Le foie est composé d'au moins six types de cellules qui sont, à côté des hépatocytes, les cellules endothéliales, épithéliales biliaires, de Kupffer, les cellules de Ito et les pit cells. Ces cellules interagissent et coopèrent à la réalisation de nombreuses fonctions métaboliques et de transport. C'est également une interaction entre les cellules de Kupffer (principalement) et les cellules de Ito qui semble être l'élément déterminant du développement des fibroses hépatiques. Enfin, dans le foie normal, des contacts spécifiques entre les hépatocytes et d'autres cellules non parenchymateuses semblent essentiels à l'expression des fonctions différenciées spécifiques.

\section{Jean Rosenbaum Philippe Mavier Daniel Dhumeaux}

\section{ADRESSE}

J. Rosenbaum : chargé de recherche à l'Inserm. $\mathrm{Ph}$. Mavier : professeur des universités, praticien hospilalier. D. Dhumeaux : professeur des universités, praticien hospilalier. Inserm U. 99, hôpital Henri-Mondor, 51, avenue du Marćchal-de-Lattre-de-Tassigny, 94010 Cré- es interactions cellulaires sont nécessaires, non seulement au fonctionnement harmonieux d'un organe où coexistent des types cellulaires différents, mais aussi à l'échelon cellulaire, où le fin réglage de multiples fonctions peut nécessiter l'intervention de signaux régulateurs provenant de l'extérieur de la cellule. Les cellules peuvent utiliser divers moyens de communication : le contact direct par l'intermédiaire de structures membranaires spécialisées ; la sécrétion de médiateurs solubles pouvant agir sur des cellules de voisinage (médiation paracrine), ou situées à distance (médiation endocrine) ; l'interaction avec la matrice extracellulaire produite par une autre cellule. Dans le foie comme dans d'autres organes, les interactions peuvent intéresser des cellules de même nature et sont dites alors homotypiques. Elles peuvent également concerner des cellules différentes les unes des autres et sont appelées hétérotypiques. L'étude des interactions homotypiques entre hépatocytes est un sujet à part entière qui ne sera pas traité ici. Le but de cette mise au point est de décrire quelques exemples d'interactions cellulaires hétérotypiques connus dans le foie adulte. La grande majorité des travaux auxquels il sera fait référence ont été effectués sur des cellules animales en culture ou, plus rarement, in vivo, chez l'animal. Il est clair que la prudence s'impose avant de les extrapoler au tissu intact d'une part, à l'homme d'autre part.

La nature même du tissu hépatique oblige à imaginer des interactions cellulaires au sein de cet organe (figures 1 et 2). Le foie adulte normal est en effet composé d'au moins six populations cellulaires résidentes distinctes.

- Les hépatocytes : ils représentent environ $70 \%$ des cellules hépatiques. Ils sont à l'origine des principaux métabolismes intra-hépatiques comme la synthèse des protéines plasmatiques, la sécrétion de la bile ou le métabolisme de nombreux composés endogènes ou exogènes (médicaments notamment).

- Les cellules épithéliales biliaires : ces cellules bordent les canaux biliaires intra-hépatiques. Certaines d'entre elles forment, avec les hépatocytes, le canal de Hering, qui 


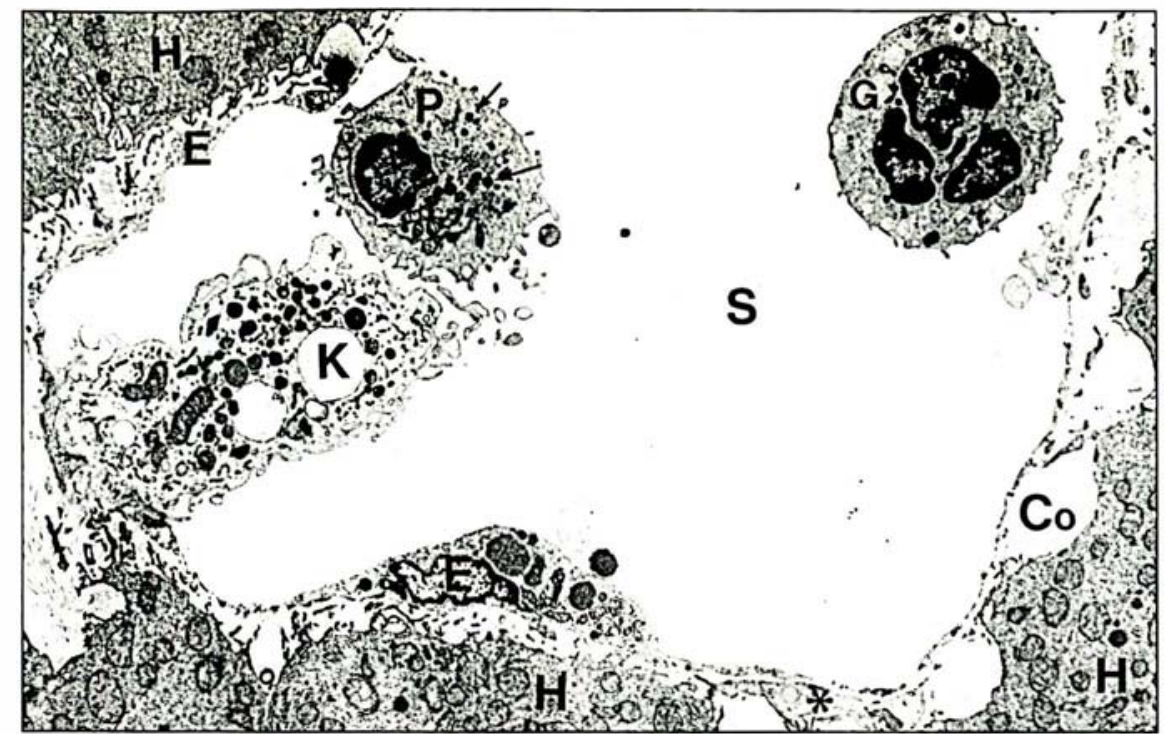

Figure 1. Aspect d'un capillaire sinusoïde hépatique humain en microscopie électronique à transmission. $E$ : cellule endothéliale; $K$ : cellule de Kupffer ; $H$ : hépatocyte ; $S$ : capillaire sinusoïde ; $P$ : pit cell lles flèches indiquent les granules caractéristiques de ces cellules); $G$ : granulocyte présent dans la lumière capillaire ; l'astérisque indique un prolongement 'd'une cellule de Ito dont le noyau n'est pas visible sur cette coupe; Co : collagène dans l'espace de Disse. (Cette photographie nous a été aimablement fournie par P. Bioulac-Sage).

transporte la bile depuis les canalicules biliaires vers les canaux biliaires interlobulaires, situés dans les espaces portes; clles sont donc en contact direct avec les hépatocytes ; elles dérivent, ainsi que ces derniers, de l'endoderme primitif, peut-être par l'intermédiaire d'un précurseur commun.

- Les cellules endothéliales : la plupart des cellules endothéliales hépatiques bordent les capillaires sinusoïdes. Ces cellules sont particulières par leur cytoplasme fenêtré et l'absence de membrane basale. Ces deux caractéristiques permettent des échanges faciles entre le sang et les hépatocytes sous-jacents à la barrière endothéliale; les cellules endothéliales dérivent du mésenchyme embryonnaire.

- Les cellules de Kupffer : ce sont des macrophages résidant dans le foie. Elles sont habituellement localisées dans la lumière sinusoïdale, au contact des cellules endothéliales, mais peuvent avoir des prolongements qui atteignent les hépatocytes $m / s=n^{\circ} 2$, col. 7 , feiricr 91
L'ensemble de ces cellules, à l'exclusion des hépatocytes, est communément désigné sous le nom de cellules non parenchymateuses. On peut rajouter à cette liste les myofibroblastes des espaces portes, les cellules musculaires lisses des parois des artérioles et les cellules nerveuses qui ont des prolongements le long des sinusoïdes.

La diversité de ces cellules et leur proximité permettent un nombre considérable d'interactions. Dans cet article, nous décrirons essentiellement des interactions mettant en cause des facteurs circulants, dans le domaine de la physiologie (métabolisme de la vitamine A, glycogénolyse, métabolisme des protéines de transport) ou dans celui de la physiopathologie hépatique (régulation de la régénération hépatique, fibrogenèse hépatique). Un exemple impliquant des contacts cellulaires et une intervention de la matrice extracellulaire scra également décrit.

\section{Métabolisme de la vitamine $A$}

Le métabolisme de la vitamine A est un exemple typique de coopération entre hépatocytes et cellules de Ito (figure 3, page 112). Le foic stocke la majorité de la vitamine A de l'organisme et plusieurs études ont montré duits de l'hématopoïèse intrahépatique fœtale, mais naissent dans le sac vitellin et colonisent le foie par voie vasculaire.

Les cellules de Ito (appelées également lipocytes, fat-storing cells ou stellate cells) : ces cellules sont localisées dans l'espace périsinusoïdal de Disse, entre hépatocytes et cellules endothéliales. Leurs très longs prolongements cytoplasmiques entourent complètement plusieurs capillaires sinusoïdes adjacents. Dans leur cytoplasme se trouvent des globules lipidiques contenant de la vitamine A. Elles ont occasionnellement des contacts étroits avec les hépatocytes.

- Enfin les pit cells, de connaissance récente, sont des lymphocytes résidents possédant des activités antitumorales de type natural killer. Ils sont visibles dans la lumière capillaire mais peuvent avoir des prolongements pénétrant la barrière endothéliale.

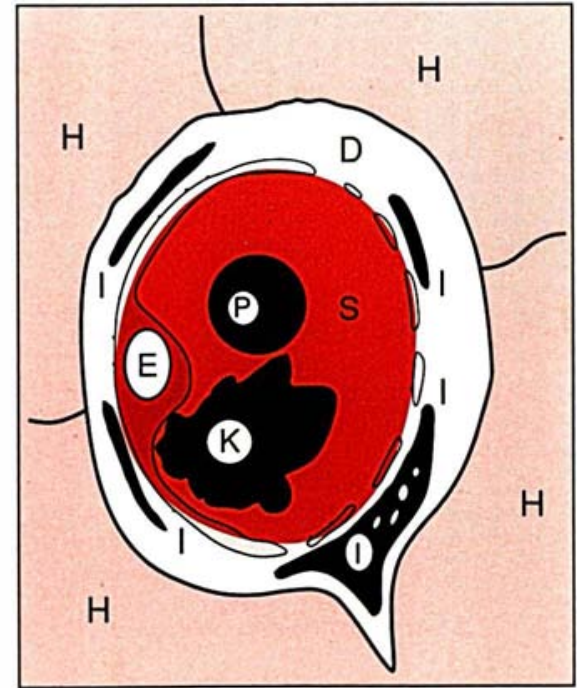

Figure 2. Schéma des différentes populations cellulaires hépatiques. Mêmes légendes que pour la figure 1 avec $D$ : espace de Disse et 1 : cellule de lto. 


\section{RÉFÉRENCES}

1. Hendriks HFJ, Elhanany E, Brouwer A, De Leeuw A, Knook DL. Uptake and processing of $\left[{ }^{3} \mathrm{H}\right]$ retinoids in rat liver studied by electron microscopic autoradiography. Hepalology $1988 ; 8$ : 276-85.

2. Blomhoff $\mathrm{R}$, Helgerud $\mathrm{P}, \mathrm{R}$ asmussen $\mathrm{M}$, Berg $\mathrm{T}$, Norum KR. In vivo uptake of chylomicron $\left[{ }^{3} \mathrm{H}\right]$ retinyl ester by rat liver : evidence for retinol transfer from parenchymal to non-parenchymal cells. Proc Nall Acad Sci USA 1982 ; 79 : 7326-30.

3. Blomhoff $R, \operatorname{Berg} T$, Norum $K R$. Transfer of retinol from parenchymal to stellate cells in liver is mediated by retinolbinding protein. Proc Natl Acad Sci USA $1988 ; 85: 3455-8$.

4. Friedman SL, Friedman G, Liu L, Boyles JK. Apolipoprotein expression by cultured rat hepatic lipocytes: analysis by polymerase chain reaction (PCR), dot blot and immunoblot. Gastroenterology $1990 ; 98$ : A 587 .

5. Hendriks HFJ, Brouwer A, Knook DL. The role of hepatic fat-storing (stellate) cells in retinoid metabolism. Hepalology $1987 ; 7$ : 1368-71.

6. Andersen KB, Nilsson A, Kvam L, Norum KR, Blomhoff R. Mobilization of retinol from Ito cells. In : Wisse $\mathrm{E}$, Knook DL, McCuskey RS, eds. Cells of the Hepalic Sinusoid, vol. 3. Rijswijk: The Kupffer Cell Foundation (sous presse).

7. Casteleijn E, Kuiper J, Van Rooij HCJ, Kamps JAAM, Koster JF, Van Berkel TJC. Endotoxin stimulates glycogenolysis in the liver by means of intercellular communication. J Biol Chem 1988 ; 263 : 6953-5.

8. Kuiper J, De Rijke YB, Zijlstra FJ, Van Waas MP, Van Berkel TJC. The induction of glycogenolysis in the perfused liver by platelet activating factor is mediated by prostaglandin $\mathrm{D}_{2}$ from Kupffer cells. Biochem Biophys Res Commun 1988; 157 : 1288-95.

9. Casteleijn E, Kuiper J, Van Rooij HCJ, Kamps JAAM, Koster JF, Van Berkel TJC. Hormonal control of glycogenolysis in parenchymal cells by Kupffer and endothelial liver cells. J Biol Chem 1988 ; 263 : que, à l'état normal, $90 \%$ de la vitamine $\mathrm{A}$ hépatique était contenu dans les granules lipidiques des cellules de Ito sous forme d'esters de rétinol. La vitamine $\mathrm{A}$ exogène, apportée par l'alimentation, est absorbée essentiellement sous forme de palmitate de rétinol, associé aux chylomicrons. Après administration de chylomicrons marqués avec du rétinol tritié, on a pu démontrer que la vitamine A était initialement captée par les hépatocytes, puis transférée dans les cellules de Ito [1, 2]. Blomhoff et al. ont observé que le transfert du rétinol vers les cellules de Ito nécessitait un couplage à la retinolbinding protein (RBP), protéine synthétisée par les hépatocytes et sécrétée dans l'espace extracellulaire [3]. Le complexe rétinol-RBP est alors reconnu par les récepteurs présents sur les cellules de Ito. La réalité de ce type de transfert in vivo est démontrée par son blocage après injection d'anticorps anti-RBP à des rats. Le mécanisme de sortie du rétinol des cellules de Ito vers ses tissus cibles est encore l'objet de discussions. Trois possibilités ont été suggérées : (a) une hypothétique sécrétion du rétinol couplé à l'apolipoprotéine $\mathrm{E}$, dont on sait qu'elle est synthétisée dans les cellules de Ito [4] ; (b) un retour obligatoire du rétinol dans les hépatocytes, qui pourrait se faire par voie transcellulaire après fixation du rétinol sur sa protéine de transport intracellulaire, la cellular retinol-binding protein [5] ; (c) une sécrétion directe dans le plasma du rétinol couplé à de la RBP néosynthétisée dans les cellules de Ito ; la capacité des cellules de Ito de rat à exprimer l'ARNm de la $\mathrm{RBP}$, à la sécréter en culture et à libérer des complexes $\left[{ }^{3} \mathrm{H}\right]$-rétinol$\mathrm{RBP}$ après incubation avec du $\left[{ }^{3} \mathrm{H}\right]-$ rétinol favorise largement cette hypothèse [6].

\section{Glycogénolyse}

L'hypothèse que les cellules sinusoïdales puissent être impliquées dans la

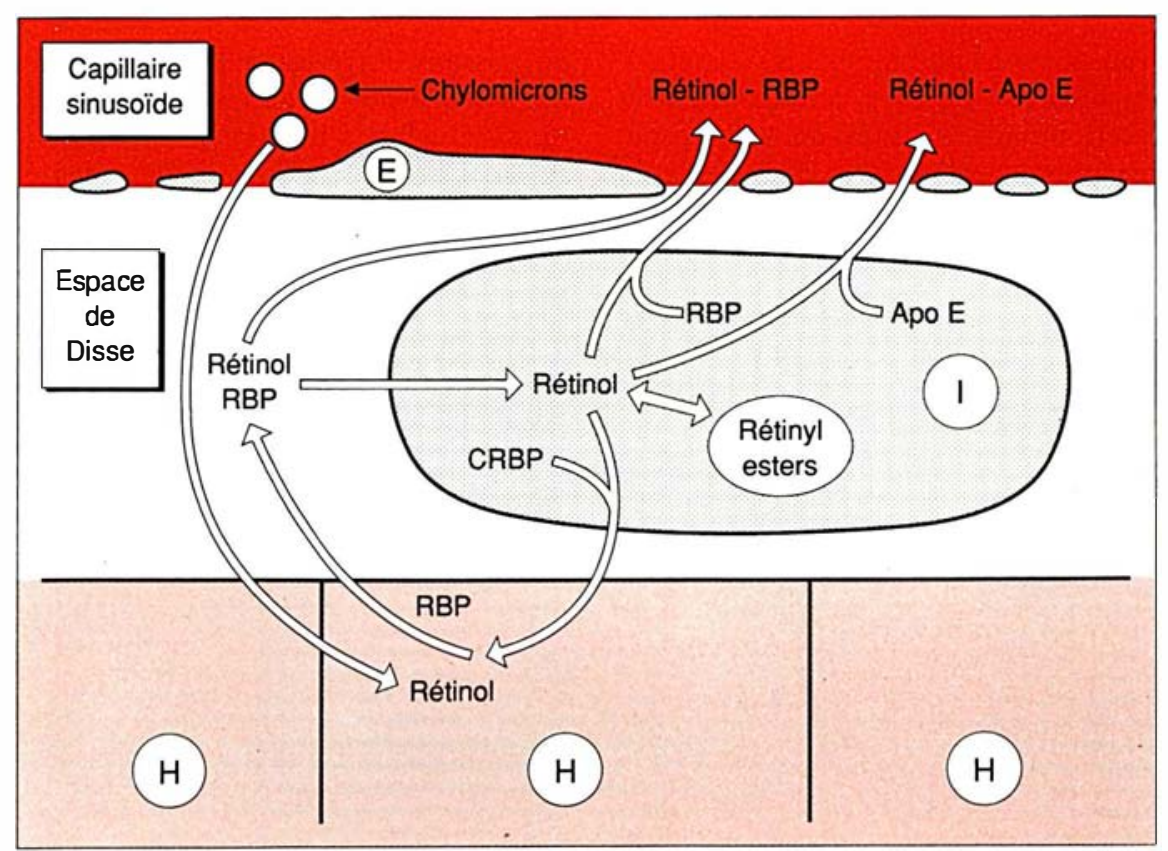

Figure 3. Métabolisme de la vitamine A dans le foie. Le rétinol apporté par les chylomicrons pénètre dans les hépatocytes $(H) d^{\prime}$ où il est sécrété lié à la retinol-binding protein (RBP). Une partie de ce complexe se fixe sur des récepteurs au niveau des cellules de Ito (I) dans lesquelles le rétinol est alors stocké. Les trois hypothèses de mobilisation du rétinol à partir des cellules de lto sont représentées: sécrétion directe par couplage à la RBP ou à l'apoprotéine $E$, ou retour vers les hépatocytes, lié à la cellular RBP (CRBP); $E$ : cellule endothéliale. 


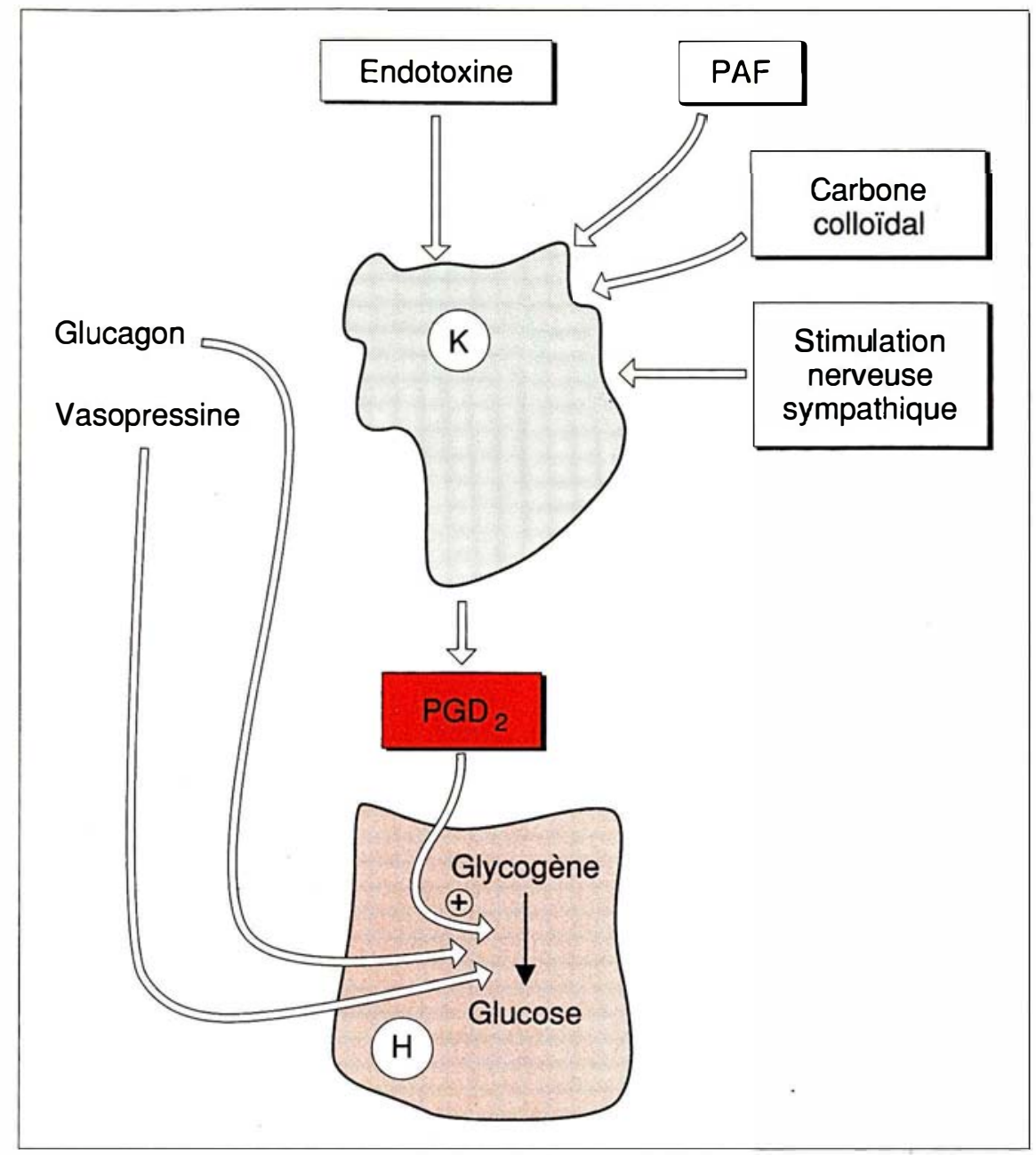

Figure 4. Rôle des cellules de Kupffer dans la glycogénolyse. En réponse à une stimulation par l'endotoxine ou le platelet-activating factor (PAF), une stimulation nerveuse sympathique ou une injection de carbone colloidal, les cellules de Kupfer (K) sécrètent la prostaglandine $D_{2}\left(P G D_{2}\right)$ qui active la glycogénolyse dans les hépatocytes $(H)$. A l'inverse, le glucagon ou la vasopressine ont un effet direct sur les hépatocytes.

médiation de l'effet glycogénolytique de divers agonistes, comme l'endotoxine ou le platelet activating factor (PAF), est dérivée de l'observation suivante: alors que des agents comme le glucagon ou la vasopressine induisent chez le rat un effet glycogénolytique aussi bien sur foie isolé perfusé que sur une préparation d'hépatocytes isolés, l'endotoxine et le PAF, actifs sur le foie total, sont sans effet sur hépatocytes isolés [7, 8]. Cette constatation a été à l'origine d'une suite de travaux du groupe de Van Berkel. Cette équipe a montré que le milieu de culture des cellules endothéliales ou des cellules $m / s n^{\circ} 2$, vol. 7, février 91 réponse était, ici encore, abolie par les inhibiteurs de la cyclooxygénase. Enfin, le PAF stimulait in vitro la sécrétion de $\mathrm{PGD}_{2}$ par les cellules de Kuppfer et, in vivo, après administration de PAF tritié, celui-ci était retrouvé au niveau de ces cellules. L'effet de la $\mathrm{PGD}_{2}$ sur la glycogénolyse était au moins en partie expliqué par sa capacité à induire la phosphorylation de la phosphorylase b, la rendant ainsi active (figure 4). Ce rôle des prostaglandines sécrétées par les cellules de Kupffer est aussi suggéré par d'autres études qui ont montré que la glycogénolyse induite par l'injection intraportale de carbone colloïdal (un activateur des cellules de Kupffer) était inhibée par l'aspirine [10]. Enfin, il est possible que les prostaglandines sécrétées par les cellules sinusoïdales soient également impliquées dans la réponse glycogénolytique secondaire à la stimulation nerveuse sympathique [11] et, au moins partiellement, dans celle induite par l'adénosine [12].

Ces résultats sont toutefois controversés. En effet, Olson et al. n'ont pas observé d'effet de l'ibuprofene, un autre inhibiteur de la cyclooxygénase, sur la réponse glycogénolytique au PAF [13]. Ces acteurs suggèrent que l'action du PAF sur foie perfusé est liée à ses effets vasoconstricteurs et que l'activation de la phosphorylase serait secondaire à l'ischémie qui en résulte. Le mécanisme intime de l'effet vasoconstricteur du PAF dans le foie n'est pas connu. Il pourrait impliquer l'intervention de cellules vasculaires contractiles et donc l'induction de la glycogénolyse serait bien encore le fait d'une interaction cellulaire.

de Kupffer incubées en présence d'endotoxine ou de PAF stimulait l'activité glycogénolytique d'hépatocytes isolés [9]. Le traitement des cellules sinusoïdales par l'aspirine, un inhibiteur de la cyclooxygénase, bloquait la production du facteur responsable de cette activation ; parallèlement, l'effet du milieu conditionné était reproduit par l'addition de prostaglandine $\mathrm{D}_{2}\left(\mathrm{PGD}_{2}\right)$. Le rôle de second messager de la $\mathrm{PGD}_{2}$ était confirmé par deux résultats: après injection d'endotoxine ou de PAF dans le perfusat du foie isolé de rat, le pic hyperglycémique était précédé par un pic de $\mathrm{PGD}_{2}$ et la

\section{Protéines de transport}

Le mécanisme de la captation du fer par les hépatocytes n'est pas complètement éclairci. Deux protéines de transport du fer, la ferritine et la transferrine, sont probablement impliquées. Dans les deux cas, ce métabolisme fait intervenir des interactions entre les hépatocytes et les cellules sinusoïdales.

Le mécanisme passant par la ferritine fait intervenir les cellules de Kupffer. Celles-ci phagocytent les érythrocytes vieillis et relarguent le fer héminique 


\section{RÉFÉRENCES}

10. Cowper KB, Currin RT, Dawson JL, Lindert KA, Lemasters JJ, Thurman RG. A new method to monitor Kupffer-cell function continuously in the perfused rat liver. Dissociation of glycogenolysis from particule phagocytosis. Biochem J 1990 ; 266 : 141-7.

11. Iwai M, Gardemann A, Puschel G, Athari A, Ulken V, Jungermann K. Communication from non-parenchymal to parenchymal cells via prostaglandins, a step in the action of sympathetic nerves on glucose and lactate balance and flow in perfused liver. In : Wisse E, Knook DL, Decker K, eds. Cells of the Hepatic Sinusoid, vol. 2. Rijswijk: The Kupffer Cell Foundation, 1989 : 251-2

12. Vom Dahl S, Wettste in M, Gerok W, Haussinger D. Simulation of release of prostaglandin D2 and thromboxane B2 from perfused rat liver by ex tracellular adenosine. Biochem J 1990 ; 270 : 39-44.

13. Lapointe DS, Olson MS. Platelet activating factor-stimulated hepatic glycogenolysis is not mediated through cyclooxygenasederived metabolites of arachidonic acid. $J$ Biol Chem 1989 ; 264 : 12130-3.

14. Sibille JC, Kondo H, Aisen D. Interactions between isolated hepatocytes and Kupffer cells in iron metabolism : a possible role for ferritin as an iron carrier prote in. Hepalology $1988 ; 8: 296-301$.

15. Tavassoli $\mathbf{M}$, Kishimoto $\mathrm{T}$, Soda $\mathrm{R}$ Kataoka M, Harjes K. Liver endothelium mediates the uptake of iron-transferrin complex by hepatocytes. Exp Cell Res 1986 ; 165 : 369-79.

16. Irie $\mathrm{S}$, Kishimoto $\mathrm{T}$, Tavassoli $\mathrm{M}$ Desialation of transferrin by rat liver endothelium. J Clin Invest $1988 ; 82$ : 508-13.

17. Tavassoli $\mathrm{M}, \mathrm{Kishimoto} \mathrm{T}$, Kataoka M. Liver endothelium mediates the hepatocyte's uptake of ceruleoplasmin. $J$ Cell Biol 1986 ; 102 : 1293-303.

18. Soda R, Tavassoli M, Jacobsen DW. Receptor distribution and the endothelial uptake of transcobalamin II in liver cell suspensions. Blood 1985 ; 65 : 795-802.

19. Soda R, Tavassoli M. Distribution of insulin receptors in liver cell suspensions using a minibead probe. Highest density on endothelial cells. Exp Cell Res $1983 ; 145$ : fixé à la ferritine. La ferritine est alors fixée sur les hépatocytes, internalisée et dégradée, libérant son fer [14]. Cette voie pourrait être la plus importante quantitativement en raison du grand nombre d'atomes de fer portés par la ferritine.

La captation hépatocytaire du fer, à partir de la transferrine plasmatique, pourrait, elle, nécessiter l'intervention préalable des cellules endothéliales sinusoïdales. Le groupe de Tavassoli a montré, à l'aide de différentes populations cellulaires hépatiques isolées de foie de rat, que seules les cellules endothéliales sinusoïdales exprimaient un récepteur pour la transferrine native. Dans une série d'élégants travaux, ils ont aussi montré que la ferrotransferrine fixée à l'endothélium était internalisée, puis libérée après passage trans-endothélial. La polarité de cette transcytose était démontrée par une étude en microscopie électronique, après perfusion de transferrine marquée à l'or colloïdal : le complexe était visible successivement sur la face luminale des cellules endothéliales, dans des vésicules endothéliales, dans l'espace de Disse, puis sur la face sinusoïdale des hépatocytes [15]. Ces auteurs ont montré qu'au cours de ce transfert, la transferrine était partiellement désialylée, ce qui : (1) lui permettait d'être reconnue par le récepteur hépatocytaire des asialoglycoprotéines ; et (2) l'empêchait de se fixer à nouveau sur l'endothélium [16].

Un mécanisme identique a été décrit pour la céruloplasmine, une glycoprotéine plasmatique qui transporte le cuivre : fixation première par les cellules endothéliales, désialylation, excrétion vers les hépatocytes [17]. Le même phénomène pourrait également s'appliquer à la transcobalamine II [18] et à l'insuline [19].

Les cellules endothéliales sont donc des intermédiaires indispensables à la fixation de ces glycoprotéines sur les hépatocytes. L'intérêt de cette médiation pourrait être de permettre aux hépatocytes d'internaliser ces différentes protéines via le même récepteur, c'est-à-dire le récepteur des asialoglycoprotéines qui reconnaît les glycoprotéines exposant un galactose terminal (démasqué par la désialylation dans les cellules endothéliales).

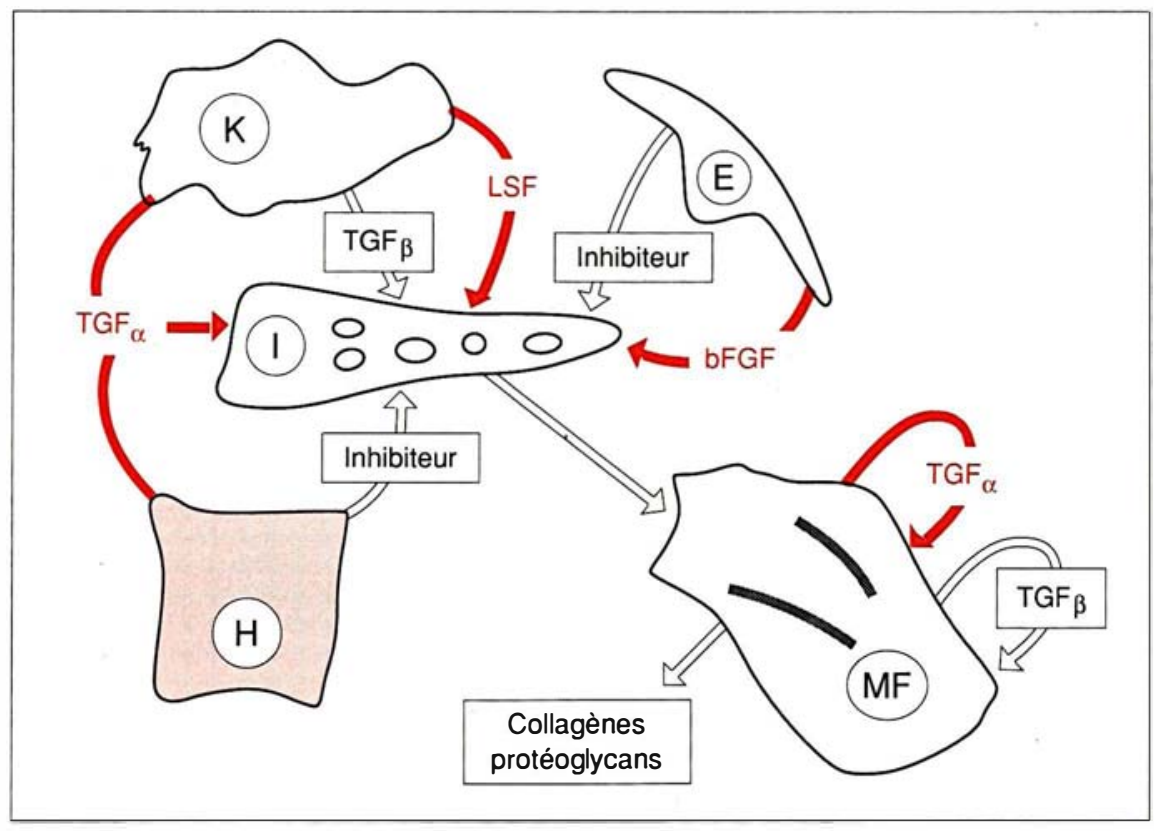

Figure 5. Mécanismes hypothétiques de l'activation des cellules de Ito. La cellule de Ito quiescente (I) reçoit des influences multiples des cellules de Kupffer $(K)$, des cellules endothéliales $(E)$ et des hépatocytes $(H)$. Certains de ces signaux peuvent provoquer la transformation myofibroblastique des cellules de lto (MF), qui sécrètent dès lors de façon autocrine des facteurs de croissance et contribuent à former la fibrose hépatique. En rouge, les influences mitogéniques. bFGF : basic fibroblast growth factor ; TGF : transforming growth factor ; LSF : lipocyte - Stimulating factor. 


\section{Fibrose hépatique, interactions concernant les cellules de Ito}

Il est actuellement clair que les cellules de Ito jouent un rôle prépondérant dans l'apparition de la fibrose hépatique. Au cours de la fibrogenèse expérimentale ainsi qu'en pathologie humaine, ces cellules perdent leur contenu en vitamine $A$, acquièrent un phénotype myofibroblastique, proliferent et déposent les constituants de la fibrose dans l'espace extracellulaire. De nombreux travaux sont consacrés aux mécanismes de l'activation et de la prolifération des cellules de Ito et de très nombreuses interactions cellulaires ont été décrites in vitro.

Plusieurs groupes ont mis en évidence, dans le milieu de culture conditionné par des cellules de Kupffer de rat, une activité mitogénique pour les cellules de Ito. Pour certains, ce facteur ne serait produit que par des cellules provenant d'animaux exposés à des traitements hépatotoxiques (tétrachlorure de carbone, galactosamine ou alcool) [20-22]; pour d'autres, ce facteur serait également libéré par les cellules de Kupffer de foie normal [23, 24]. La nature précise de ce (ou ces) facteur(s) est encore inconnue. Le groupe de Friedman a mis en évidence une molécule de très faible poids moléculaire (lipocyte-stimulating factor, LSF) qui agirait indirectement sur la prolifération des cellules de Ito en induisant, à leur surface, l'expression du récepteur du platelet-derived growth factor [24]. D'autres auteurs ont montré que les cellules de Kupffer synthétisaient du transforming growth factor $\alpha$ (TGF $\alpha$ ) qui est effectivement mitogénique pour les cellules de Ito [25]. Outre ces effets sur la prolifération, il a été aussi observé que le milieu conditionné par les cellules de Kupffer stimulait la synthèse de collagène [24] et de protéoglycans [25] par les cellules de Ito. Très récemment, Matsuoka et al. ont montré que l'augmentation de synthèse du collagène était due à la sécrétion, par les cellules de Kupffer, de transforming growth factor $\beta 1$ (TGF $\beta 1$ ). Un rôle physiopathologique possible de cette sécrétion de TGF $\beta 1$ par les cellules de Kupffer était aussi suggéré par $m / s n^{\circ} 2$, vol. 7, féurier 91 l'augmentation de l'expression de l'ARNm du TGF $\beta 1$ dans les cellules de Kupffer provenant de rats soumis à un régime conduisant à l'apparition d'une fibrose hépatique [20]. Le TGF $\beta 1$ serait aussi le médiateur responsable de l'effet des cellules de Kupffer sur la synthèse de protéoglycans par les cellules de Ito [25]. Les autres cellules hépatiques participent également à la régulation de la prolifération des cellules de Ito. Les cellules endothéliales sinusoïdales de souris ont une capacité bifonctionnelle, puisqu'elles sécrètent une protéine inhibant la prolifération des cellules de Ito [26] et que, par ailleurs, elles synthétisent le fibroblast growth factor basique (FGFb) qui est un puissant mitogène pour les cellules de Ito [27]. Enfin, les hépatocytes auraient aussi un double rôle en sécrétant un facteur inhibant la croissance des cellules de Ito chez la souris [28] et en synthétisant le TGF $\alpha$ [29].

La modulation du phénotype quiescent des cellules de Ito vers le phénotype activé est un phénomène complexe qui devrait prendre en compte au moins les mécanismes décrits ci-dessus. La quiescence de ces cellules dans le foie normal est probablement liée à la conjonction d'interactions cellules-cellules, telles que celles qui ont été exposées, et cellules-matrice extracellulaire. Le contenu en vitamine A de ces cellules pourrait également intervenir. Au cours de la fibrogenèse, leur activation (transformation phénotypique, prolifération et synthèse de composants de la fibrose) pourrait être liée à des signaux provenant des cellules de Kupffer et peut-être aussi des hépatocytes. La séquence des événements pourrait être la suivante : induction (par le TGF $\beta 1$ provenant des cellules de Kupffer ?) de la synthèse par les cellules de Ito d'une matrice extracellulaire riche en collagène de type I ; parallèlement, dégradation de la matrice physiologique, composée essentiellement de collagène de type IV, par une gélatinase sécrétée par les cellules de Ito ; ces deux événements pourraient fournir un support favorable à la prolifération des cellules de Ito, également stimulée par les facteurs de croissance provenant des cellules de voisinage; enfin, le phénomène pourrait être auto-entretenu par une production par les cellules de Ito " activées ", de TGF $\alpha$ et de TGF $\beta 1$ (figure 5, p. 114).

\section{Régénération hépatique}

La régulation de la masse hépatique est un phénomène étroitement contrôlé. Que ce soit chez l'homme ou chez le rat, après hépatectomie, le foie restant subit une hypertrophie compensatrice très rapide qui cesse lorsque la masse d'origine a été restituée (de 7 à 10 jours au total). Il est vraisemblable qu'une adaptation aussi rapide et précise fait intervenir de multiples facteurs de régulation dont l'origine peut être hépatique ou extra-hépatique. Au cours de ces dernières années, en utilisant le modèle classique d'hépatectomie des deux tiers chez le rat, plusieurs travaux se sont centrés sur le rôle de facteurs de croissance agissant sur les hépatocytes et produits soit par les hépatocytes eux-mêmes (stimulation autocrine), soit par les cellules non parenchymateuses (stimulation paracrine). La compilation de ces données aboutit à un schéma assez séduisant: (1) dès la $4^{\mathrm{e}}$ heure après l'hépatectomie, augmentation importante de l'expression de l'ARNm de l'heparin-binding growth factor 1 (HBGF 1, ou FGF acide) dans les hépatocytes et dans les cellules non parenchymateuses [30]. L'HBGF 1, qui est mitogénique pour les hépatocytes, pourrait être le premier stimulus de la régénération hépatocytaire; (2) quelques heures plus tard, coïncidant avec le pic de synthèse d'ADN dans le foie, expression hépatocytaire accrue de l'ARNm du TGF $\alpha$, un autre facteur mitogénique pour les hépatocytes [29]; (3) enfin, de 24 à 72 heures après l'hépatectomie, augmentation progressive de l'expression de l'ARNm du TGF $\beta 1$. Cette expression est limitée apparemment aux cellules endothéliales [31]. Le TGF $\beta 1$, qui inhibe la réplication hépatocytaire aussi bien in vitro qu'in vivo, jouerait ainsi un rôle dans l'arrêt de la multiplication des hépatocytes.

Le dernier-né des facteurs de croissance hépatocytaire, hepatocyte growth factor (HGF), récemment purifié et cloné [32-34], joue probablement un rôle important dans le phénomène de 
20. Matsuoka M, Zhang MY, Tsukamoto $\mathrm{H}$. Sensitization of hepatic lipocytes by high fat diet to stimulatory effects of Kupffer cell-derived factors : implication in alcoholic liver fibrogenesis. Hepalology 1990 ; $11: 173-82$

21. Armendariz-Borunda J, Greenwel P, Rojkind M. Kupffer cells from CCl4 - treated rat livers induce skin fibroblast and liver fat-storing cell proliferation in culture. Matrix $1989 ; 150-8$.

22. Shiratori $\mathrm{Y}$, Geerts $\mathrm{A}$, Ichida $\mathrm{T}$, Kawase T, Wisse E. Kupffer cells from $\mathrm{CCl}^{-}$- induced fibrotic livers stimulate proliferation of fat-storing cells. $J$ Hepatol 1986 ; $3: 294-303$

23. Zerbe O, Gressner AM. Proliferation of fat-storing cells is stimulated by secretions of Kupffer cells from normal and injured liver. Exp Mol Pathol 1988; 49: 87-101.

24. Friedman SL, Arthur MJP. Activation of cultured rat hepatic lipocytes by Kupffer cell conditioned medium. Direct enhancement of matrix synthesis and stimulation of cell proliferation via induction of plateletderived growth factor receptors. $J$ Clin Invest 1989 ; 84: 1780-5.

25. Meyer DH, Bachem MG, Gressner AM. Modulation of hepatic lipocyte proteoglycan synthesis and proliferation by Kupffer cell-derived transforming growth factors type $\beta 1$ and type $\alpha$. Biochem Biophys Res Commun 1990; 171: 1122-9.

26. Rosenbaum J, Mavier P, Preaux AM, Lescs MC, Dhumeaux D. Mouse hepatic endothelial cells in culture secrete a growth inhibitor for hepatic lipocytes and Balb/c 3T3 fibroblasts. J Hepalol 1989; 9 295-300.

27. Rosenbaum J, Mavier P, Preaux AM, Dhumeaux D. Demonstration of a basic fibroblast growth factor-like molecule in mouse hepatic endothelial cells. Biochem Biophys Res Commun 1989 ; 164 : 1099-104.

28. Chen W, Steffan AM, Braunwald J, Nonenmacher $\mathrm{H}$, Kirn A, Gendrault JL. Inhibition in fat-storing cell multiplication by a factor produced by normal cultured murine hepatocytes. J Hepalol 1990 ; 11 : 330-8.

29. Mead JE, Fausto N. Transforming growth factor may be a physiological regulator of liver regeneration by means of an autocrine mechanism. Proc Nall Acad Sci USA 1989; 86 : 1558-62.

30. Kan M, Huang J, Mansson PE, Yasumitsu H, Carr B, McKeehan WL Heparin-binding growth factor type 1 (acidic fibroblast growth factor): a potential of hepatocyte proliferation. Proc Nall Acad Sci USA 1989 ; $86: 7432-6$.

31. Braun L, Mead JE, Panzica M, Mikumo R, Bell GI, Fausto N. Transforming growth factor $\beta$ mRNA increases during liver regeneration: a possible paracrine mechanism of growth regulation. Proc Nall Acad Sci USA 1988 ; 85 : 1539-43.

32. Miyazawa K, Tsubouchi H, Naka D, et al. Molecular cloning and sequence analysis of cDNA for human hepatocyte growth factor. Biochem Biophys Res Commun 1989 ; 163: 967-73.

33. Nakamura $T$, Nishizawa $T$, Hagiya $M$, et al. Molecular cloning and expression of human hepatocyte growth factor. Nalure $1989 ; 342: 440-3$

34. Zarnegar R, Muga S, Rahija R, Michalopoulos G. Tissue distribution of hepatopoietin A : a heparin-binding polypeptide growth factor for hepatocytes. Proc Nall Acad Sci USA 1990 ; 87 : 1252-6.

35. Lindroos $\mathrm{P}$, Zarnegar R, Michalopoulos G. Hepatocyte growth factor (HGF, HPTA) regulates liver regeneration via both endocrine and autocrine mechanisms. Hepalology $1990 ; 12: 913$.

36. Kinoshita $T$, Tashiro $K$, Nakamura $T$. Marked increase of HGF mRNA in nonparenchymal liver cells of rats treated with hepatotoxins. Biochem Biophys Res Commun $1989 ; 165: 1229-34$.

37. Hirono S, Tsubouchi H, Morita Y, et al. In situ hybridization of human hepatocyte growth factor (HGF) mRNA and hHGF activity in the liver and spleen of Japanese monkey. Hepalology 1990 ; 12 : 913.

38. Fraslin JM, Kneip B, Vaulont $\mathrm{S}$, Glaise D, Munnich A, GuguenGuillouzo C. Dependence of hepatocytespecific gene expression on cell-cell interactions in primary culture. EMBO J 1985 ; $4: 2487-91$

39. Guguen-Guillouzo C. Rôle des interactions cellulaires homotypiques et hétérotypiques dans l'expression des fonctions spécifiques par les hépatocytes en culture. In Guillouzo A, Guguen-Guillouzo C, eds. Hépalocyles isolés el en culture. Paris: Inserm/John Libbey Eurotext, 1986: 271-96.

40. Morin O, Normand C. Long term maintenance of hepatocyte functional activity in co-culture : requirements for sinusoidal endothelial cells and dexamethasone. J Cell Physiol 1986 ; 129 : 103-10.

41. Michalopoulos G, Russel F, Biles C. Primary cultures of hepatocytes on human fibroblasts. In vitro 1979 ; 15 : 789-806. régénération, encore que des divergences persistent quant à son mécanisme d'action, endocrine, paracrine ou autocrine. Après hépatectomie des deux tiers, le pic sérique très précoce de HGF serait d'origine extrahépatique [35]. En revanche, après administration de tétrachlorure de carbone, HGF serait produit dans le foie ; dans cet organe, si un immunomarquage positif a été mis en évidence dans les hépatocytes [35], l'ARNm de HGF n'a été détecté que dans les cellules non parenchymateuses [36, 37], indiquant qu'elles sont probablement responsables de sa synthèse.

\section{Maintien de la différenciation des hépatocytes en culture}

Les hépatocytes isolés maintenus en culture perdent très rapidement leurs fonctions différenciées, comme en témoignent la diminution de la sécrétion d'albumine et la baisse en quelques heures ou jours de la transcription des gènes spécifiques [38]. Les interactions homotypiques recréées en culture sont donc insuffisantes au maintien de l'état différencié. Le fait que des interactions hétérotypiques soient nécessaires pour maintenir cette différenciation hépatocytaire in vitro a été évoqué par plusieurs auteurs, notamment par l'équipe de C. Guguen-Guillouzo (Inserm U. 49, Rennes, France) dans un modèle de co-culture composé d'hépatocytes de rat ou d'homme ainsi que d'une lignée de cellules épithéliales biliaires isolées de foie de rat (cellules probablement dérivées des cellules bordant le canal de Hering et donc, in vivo, en contact avec les hépatocytes). Dans ce modèle, les hépatocytes maintenaient leurs fonctions différenciées pendant plusieurs mois avec, notamment, production d'albumine, de protéines et de réaction inflammatoire et maintien des systèmes enzymatiques de biotransformation des médicaments [39]. Lorsque les cellules épithéliales biliaires étaient ajoutées tardivement à la culture d'hépatocytes, on observait la réexpression des fonctions différenciées qui avaient été perdues. Ces auteurs ont démontré que le maintien des fonctions de 
l'hépatocyte était lié, au moins en partie, à la persistance - à un haut niveau de la transcription - des gènes correspondants. Le signal responsable de ce phénomène dans le modèle de co-culture n'est pas encore complètement élucidé. Il ne s'agit pas d'un médiateur soluble produit par les cellules biliaires car le milieu conditionné par ces cellules est inefficace. Les deux points caractéristiques de la co-culture sont la formation très rapide de contacts étroits entre les deux types cellulaires et l'apparition secondaire d'un matériel extracellulaire dense formé essentiellement de fibronectine mais aussi de collagène de type IV, de laminine et d'autres types de collagène. L'hypothèse la plus probable est que le signal induit par contact entre les deux types cellulaires fait intervenir une ou plusieurs protéines membranaires qui restent à caractériser et que la matrice extracellulaire intervient secondairement pour stabiliser le système.

L'effet des cellules biliaires n'est cependant pas spécifique. D'autres auteurs ont reproduit des résultats analogues en co-cultivant des hépatocytes de rat avec une lignée de cellules endothéliales sinusoïdales homologues [40]. L'addition de cellules endothéliales d'aorte bovine [40], ou de fibroblastes [41], n'avait qu'un effet transitoire. Il est donc possible que différentes cellules puissent pourvoir le signal adéquat, mais une spécificité d'organe pourrait être nécessaire.

\section{Conclusion}

Bien d'autres interactions ont été décrites entre les cellules hépatiques, comme l'effet des cellules de Kupffer sur la synthèse protéique des hépatocytes et les interactions entre pit cells et cellules de Kupffer... L'objet de cette mise au point, obligatoirement limitée, était de mettre en évidence l'interdépendance majeure entre les différentes cellules qui composent le foie. A cet égard, il faut souligner le rôle important des cellules non parenchymateuses, longtemps négligées, et dont on sait maintenant qu'elles interviennent dans des métabolismes "typiquement " hépatocytaires

$\mathrm{m} / \mathrm{s} n^{\circ} 2$, vol. 7, février 91

\section{Summary}

Cellular interactions in the liver

The liver is composed of six main cellular types : hepatocytes, endothelial cells, Kupffer cells, Ito cells, biliary epithelial cells and pit cells. This article describes some of the interactions which occur between these cells. Vitamin A, coming from the portal vein, enters the hepatocytes; it is then secreted as a complex with retinolbinding protein which binds to Ito cells where vitamin A is finally stored. Kupffer cells are involved in the glycogenolytic effect of endotoxin and platelet-activating factor via secretion of prostaglandin $\mathrm{D}_{2}$ which activates phosphorylase $\mathrm{b}$ in hepatocytes. Native ferrotransferrin cannot bind to hepatocytes but must transit through endothelial cells where it is partly desialylated, becoming then a ligand for the hepatocyte asialoglycoprotein receptor. Ito cells are exposed to mitogenic and profibrogenic signals originating mainly from Kupffer cells ; these effects could be counter-balanced by molecules secreted by endothelial cells and hepatocytes. Following 2/3 hepatectomy, hepatocyte proliferation is stimulated by growth factors synthetized by non-parenchymal cells (and hepatocytes themselves) ; this proliferation could be put to a stop by endothelial cell-derived transforming growth factor $\beta 1$. Cultured hepatocytes rapidly lose their differentiated functions ; when cocultured with liver epithelial cells, they can maintain these functions for several weeks, probably due to interactions involving membrane proteins.

\section{TIRÉS A PART}

J. Rosenbaum. 